\title{
Nuclear spin dynamics in parabolic quantum wells
}

\author{
Ionel Ţifrea $\left.{ }^{*}\right]$ Michael E. Flatté \\ Department of Physics and Astronomy, University of Iowa, Iowa City 52242, USA
}

(Dated: November 20, 2018)

\begin{abstract}
We present a detailed analytical and numerical analysis of the nuclear spin dynamics in parabolic quantum wells. The shallow potential of parabolic quantum wells permits substantial modification of the electronic wave function in small electric fields. The nuclear spin relaxation via the hyperfine interaction depends on the electronic local density of states, therefore the local nuclear relaxation time depends sensitively on the electric field. For an inhomogeneous nuclear magnetization, such as generated by dynamic nuclear polarization, the total nuclear magnetization dynamics can similarly be altered. We examine this effect quantitatively and the effect of temperature, field, well thickness, and nuclear spin diffusion.
\end{abstract}

\section{INTRODUCTION}

The long electronic spin coherence times in GaAs structures [1] may assist the practical realization of spin based electronics, including quantum information processing.[2] Electronic spin interactions with phonons, nuclear spins, other electron spins and impurities will limit the value of the electronic spin relaxation and coherence times. Due to much longer coherence times nuclear spins can also be useful elements of spin-based devices. Compared to electronic spin relaxation times, of the order of $100 \mathrm{~ns}$, nuclear spin relaxation times in the absence of carriers are roughly $10 \mathrm{~min}$ in GaAs structures.[3-5] Yet the local electronic density of states near a nuclear spin can be increased to the point where the hyperfine interaction dominates nuclear spin relaxation and the relaxation times are shorter than $1 \mathrm{~s}$.

In this work we investigate the nuclear spin relaxation time in a parabolic quantum well (PQW). In this low dimensional structure electrons are trapped in a parabolic potential that restricts their motion along one direction. Such a profile for the confining potential can be obtained by gradually varying the $\mathrm{Al}$ concentration, $x$, of $\mathrm{Al}_{x} \mathrm{Ga}_{1-x}$ As layers between two barriers. [6] In the presence of an electric field the minimum of the confining potential is displaced with respect to the center of the PQW, whereas the shape of the confining potential and implicitly of the envelope wave function are largely conserved. This particular property of the PQW makes it suitable for the practical realization of electronic devices based on the electrical manipulation of the electronic or nuclear spins. Recent experiments[7] demonstrated that considerable electrical control of electronic spin dynamics ( $g$-factors) can be achieved in shallow potential parabolic quantum wells.

The standard technique used to extract nuclear spin relaxation times is nuclear magnetic resonance (NMR) spectroscopy. For low dimensional systems such as PQW's the signal in a standard NMR experiment from the number of available nuclei across the sample is too small to measure. The acquired polarization of nuclei in such samples under a magnetic field of $10 \mathrm{~T}$ is about $10^{-4}-10^{-5}$ per nucleus, insufficient to gener- ate signal from a sample with $10^{12}-10^{15}$ nuclei.[4] Higher levels of nuclear polarization are required for a detectible NMR signal. An alternative to standard NMR spectroscopy is optically pumped NMR spectroscopy. In this approach non-equilibrium electron spin polarizations are generated through exciting electronic interband transitions. These spin-polarized electrons then polarize the nuclei during their relaxation. $[4,8-10]$ This process is known as dynamical nuclear polarization (DNP).[11] DNP has recently been achieved electrically in ferromagnetic-semiconductor heterostructures.[12]

The nuclear spin coherence and relaxation dynamics will also be influenced by the fact that all nuclei in GaAs carry spins, so nuclear diffusion cannot be ignored.[9, 13] In the DNP process the initial spatial distribution of the nuclear polarization will follow the nuclear-electron mutual spin-flip rate, proportional to the square of the electronic local density of states, for the hyperfine interaction is a point contact interaction. In lightly doped bulk semiconductors the initial polarization of the nuclei is realized around impurities, whereas in PQW's the confining potential creates a position-dependent electron distribution, which will determine also the initial polarization of the nuclei through the sample. Under continual optical pumping the diffusion process will lead, eventually, to the uniform polarization of the nuclei in the whole sample. The value of the diffusion constant in GaAs systems was estimated previously by Paget[13] for bulk samples and by Malinowski and Harley[9] for square quantum well structures to be of the order of $10^{-14}-10^{-13} \mathrm{~cm}^{2} / \mathrm{s}$.

Once polarized, the nuclear spin dynamics can be controlled directly with magnetic fields, or indirectly with electric fields. A method for electric field control of the nuclear spin relaxation time in quantum well (QW) structures was first reported by Smet et al.[5], and is based on electrically tuning the electron density in a QW across a Quantum Hall ferromagnet transition. In the low temperature regime such control is possible due to electric field induced modifications to the spectrum of collective excitations.

In this work we analyze two different situations in which the nuclear spin relaxation time can be controlled with an external electric field. We treat the twodimensional electron gas at much higher temperatures, 
outside of the Quantum Hall regime. The DNP process remains very efficient up to $30 \mathrm{~K}$, and we predict a sensitive dependence of the nuclear spin relaxation time on the electric field, originating from the manipulation of the electronic local density of states across the PQW. This manipulation of the electronic local density of states produces a position-dependent nuclear spin relaxation rate. The position dependence can be observed in a sample with a single $\delta$-doped layer of an NMR active material inserted at a certain position inside the PQW. The inserted layer needs to be made of different nuclei than the one present in the host PQW to ensure that its observed resonant frequency is not the same as that of the host PQW nuclei. Even in a sample without a specific $\delta$-doped layer substantial control of the nuclear relaxation rate is possible. For uniformly distributed nuclei in the PQW, the appropriately averaged nuclear relaxation time depends on the occupancy factor of the electronic conduction subbands, decreasing stepwise as the electron density increases.

We report both analytical and numerical results for the electric field dependent nuclear spin relaxation time. The special structure of the confining potential in the PQW allows exact analytical solutions for the position dependent electronic density across the structure. More accurate results can be obtained from numerical calculations of the electronic local density of states in the PQW based on a $\mathbf{k} \cdot \mathbf{p}$ code, however we find that the analytic results are respectably accurate for the structures we considered. We find that the electron and nuclear spin relaxation times can be tuned over a range of at least an order of magnitude using an external electric field. To identify the most suitable PQW structure for the nuclear spin relaxation time manipulation, we investigate two different possible samples, with different confining potentials.

The paper is organized as follows. The second section is dedicated to the general presentation of the calculation of the nuclear relaxation time as a result of the hyperfine interaction. The third section presents our analytical and numerical analysis of the PQW. Finally we summarize our results in the last section.

\section{THE GENERAL FORMALISM FOR THE ELECTRONIC AND NUCLEAR RELAXATION TIMES}

Our analysis of the electronic and nuclear spin relaxation times in low dimensional systems follows the previous calculation by Overhauser[14] for the case of three dimensional (3D) bulk metals. We only consider effects due to the hyperfine interaction which couple the electronic and nuclear spins. The interaction Hamiltonian, known also as the Fermi contact term, can be written as

$$
H=\sum_{n} H\left(\mathbf{r}_{n}\right)=\sum_{n} \frac{8 \pi}{3} \beta_{e} \beta_{n}\left(\vec{\sigma}_{n} \cdot \vec{\sigma}_{e}\right) \delta\left(\mathbf{r}-\mathbf{r}_{n}\right),
$$

where $\beta_{n}$ and $\beta_{e}$ are the nuclear and electron spin magnetic moments, $\vec{\sigma}_{n}$ and $\vec{\sigma}_{e}$ the Pauli spin operators for the nucleus and electron, and $\delta(x)$ is the usual delta function. The argument of the delta function in Eq. (2.1), $\mathbf{r}-\mathbf{r}_{n}$, represents the relative distance between the nuclear and electronic spins, and shows the local character of the hyperfine interaction. The effects of the Fermi contact term are better understood if we express the product of the two Pauli spin operators, $\left(\vec{\sigma}_{n} \cdot \vec{\sigma}_{e}\right)$, in terms of the creation and annihilation operators, $\sigma_{n(e)}^{ \pm}$, as

$$
\vec{\sigma}_{n} \cdot \vec{\sigma}_{e}=\sigma_{n}^{z} \sigma_{e}^{z}+2\left(\sigma_{n}^{+} \sigma_{e}^{-}+\sigma_{n}^{-} \sigma_{e}^{+}\right),
$$

an expression which clearly identifies the spin-flip processes involving both the electron and nuclear spin. The second term in Eq. (2.2) flips a nuclear spin from down to up in a process which implies also an electron spin flip from up to down. The last term in Eq. (2.2) describes the reverse process.

To understand the spin relaxation process one has to consider the effects of a magnetic field $\mathcal{H}$ acting on the particle's spin. The final result of the interaction between the spin and the magnetic field will be an orientation of the spin, parallel or antiparallel, relative to the applied field. However, such a process does not occur instantaneously, and its time dependence defines the characteristic spin relaxation time. The dependence on different parameters of the relaxation time originates from the particular mechanism which produces the spin alignment. In our case this mechanism will be the hyperfine interaction. As the Fermi contact term involves both the electron and the nuclear spins we expect to obtain a single equation which will determine both the electron and nuclear spin relaxation times.

The approximations we introduce are natural ones for nanostructures. We consider the electronic system to be in spin equilibrium with itself. Thus even though the electronic local density of states may vary from position to position, the electronic spin polarization is the same everywhere. Situations where the electronic spin system has position-dependent spin polarization can be considered by combining the types of expressions found here with electron spin diffusion equations. For our purposes here we assume that at any specific time we have $N_{+}$of the electrons with their spins oriented along the applied field (spin up) and $N_{-}$with their spins oriented antiparallel to the applied field (spin down).

In contrast to the electronic system we permit the nuclear system to develop spatially-dependent polarization. For simplicity we will derive the expressions for electronic and nuclear spin relaxation times considering only nuclei with total spin $1 / 2$; the generalization for other nuclear spin values is straightforward. Thus for a given nucleus the probability of being spin up is $M_{+}\left(\mathbf{r}_{n}\right)$ and of spin down is $M_{-}\left(\mathbf{r}_{n}\right)$. One might be concerned if these local polarizations are well defined - it is known that in some circumstances non-Markovian effects can play a role, and we ignore such effects here. This approximation can be 
interpreted in some circumstances as averaging over sufficiently long times, and in other circumstances of coarsegraining the nuclear system over a volume large compared to the internuclear distance, but small compared to relevant electronic length scales. This coarse-graining permits us to replace the orientation of a given nucleus in these equations with a statistical average.

The time dependence of the relaxation process will be related to the time evolution of both the total electronic magnetization and the position-dependent nuclear magnetization, which can be expressed, in terms of the differences, $D=N_{+}-N_{-}$, and $\Delta\left(\mathbf{r}_{n}\right)=M_{+}\left(\mathbf{r}_{n}\right)-M_{-}\left(\mathbf{r}_{n}\right)$. If we denote by $W_{+-}\left(\mathbf{r}_{n}\right)$ the total numbers of electron spins which flip from down to up per second, and by $W_{-+}\left(\mathbf{r}_{n}\right)$ the total number of electron spins which flip from up to down per second, each from interaction with nucleus $n$, we can write the change in $D$ as

$$
\frac{d D}{d t}=2 \sum_{n}\left[W_{+-}\left(\mathbf{r}_{n}\right)-W_{-+}\left(\mathbf{r}_{n}\right)\right],
$$

and in $\Delta\left(\mathbf{r}_{n}\right)$ as

$$
\frac{d \Delta\left(\mathbf{r}_{n}\right)}{d t}=2\left[W_{-+}\left(\mathbf{r}_{n}\right)-W_{+-}\left(\mathbf{r}_{n}\right)\right]
$$

The evaluation of $W_{+-}\left(\mathbf{r}_{n}\right)$ and $W_{-+}\left(\mathbf{r}_{n}\right)$ will be done based on Fermi's golden rule by treating the hyperfine interaction as a time dependent perturbation.

Consider an electron transition between two states $\mathbf{k}_{\downarrow}$ and $\mathbf{k}_{\uparrow}^{\prime}$ in which the electron spin is flipped from down to up. According to Fermi's golden rule, the electronic transition probability from state $\mathbf{k}$ to state $\mathbf{k}^{\prime}$ induced by nucleus $n$ is given by

$$
W_{\mathbf{k k}^{\prime}}^{\downarrow \uparrow}\left(\mathbf{r}_{n}\right)=\frac{2 \pi}{\hbar}\left|H_{\mathbf{k k}^{\prime}}\left(\mathbf{r}_{n}\right)\right|^{2} N_{e}\left(\mathbf{k}_{\uparrow}^{\prime}\right) \delta\left(E_{i}-E_{f}\right),
$$

where $H_{\mathbf{k k}^{\prime}}\left(\mathbf{r}_{n}\right)=\left\langle\psi_{f}\left(\mathbf{k}_{\uparrow}^{\prime}\right)\left|H\left(\mathbf{r}_{n}\right)\right| \psi_{i}\left(\mathbf{k}_{\downarrow}\right)\right\rangle$ represents the matrix elements of the hyperfine interaction Hamiltonian for nucleus $n, N_{e}\left(\mathbf{k}_{\uparrow}^{\prime}\right)$ is the electronic density of states for the final state, and $E_{i}$ and $E_{f}$ are the energies corresponding to the initial and final state. Accordingly, the total number of spin flips per second induced by nucleus $n, W_{+-}\left(\mathbf{r}_{n}\right)$, is

$$
\begin{aligned}
& W_{+-}\left(\mathbf{r}_{n}\right)= \\
& \sum_{\mathbf{k}} W_{\mathbf{k k}^{\prime}}^{\downarrow \uparrow}\left(\mathbf{r}_{n}\right) M_{+}\left(\mathbf{r}_{n}\right) f_{F D}\left(\mathbf{k}_{\downarrow}, E_{i}\right)\left[1-f_{F D}\left(\mathbf{k}_{\uparrow}^{\prime}, E_{f}\right)\right],
\end{aligned}
$$

where $f_{F D}\left(\mathbf{p}_{\sigma}, E\right)$ is the usual Fermi-Dirac distribution function for an electron with momentum $\mathbf{p}$ and spin $\sigma$. The energy conservation expression

$$
\frac{\mathbf{k}_{\uparrow}^{\prime 2}}{2 m}-\beta_{e} \mathcal{H}-\beta_{n} \mathcal{H}=\frac{\mathbf{k}_{\downarrow}^{2}}{2 m}+\beta_{e} \mathcal{H}+\beta_{n} \mathcal{H},
$$

allows us to eliminate $\mathbf{k}_{\uparrow}^{\prime}$ and estimate $W_{+-}\left(\mathbf{r}_{n}\right)$ using Eq. (2.6) by replacing the sum over the momenta with an integration over the energy using the density of states. The integration over energies can be performed simply in the case of small magnetic fields, $\beta_{e(n)} \mathcal{H} \ll k_{B} T$ ( $T$ is the temperature), for which the Fermi-Dirac function can be expanded in a Taylor series. $W_{-+}\left(\mathbf{r}_{n}\right)$ is calculated in a similar way using $M_{-}\left(\mathbf{r}_{n}\right)$ instead of $M_{+}\left(\mathbf{r}_{n}\right)$ and changing $\mathcal{H}$ to $-\mathcal{H}$.

After some algebra we find that the time dependence of the electron magnetization is described by the following equation

$$
\begin{aligned}
& \frac{d D}{d t}=\sum_{n} \frac{512 \pi^{3} \beta_{n}^{2} \beta_{e}^{2} \tilde{N}_{e}\left|\psi\left(\mathbf{r}_{n}\right)\right|^{4}}{9 \hbar V}\left(D_{0}-D\right) \\
& +\sum_{n} \frac{1024 \pi^{3} \beta_{n}^{2} \beta_{e}^{2} \tilde{N}_{e}^{2} k_{B} T\left|\psi\left(\mathbf{r}_{n}\right)\right|^{4}}{9 \hbar}\left(\Delta_{0}\left(\mathbf{r}_{n}\right)-\Delta\left(\mathbf{r}_{n}\right)\right),
\end{aligned}
$$

where $\tilde{N}_{e}=\int d \varepsilon N_{e}(\varepsilon) f_{F D}^{\prime}(\varepsilon), D_{0}$ and $\Delta_{0}\left(\mathbf{r}_{n}\right)$ are the thermal equilibrium values for $D$ and $\Delta\left(\mathbf{r}_{n}\right)$, respectively, and $\psi\left(\mathbf{r}_{n}\right)$ is the electronic wave function at the $n$ 'th nuclear position. The first term on the right hand side (rhs) of Eq. (2.8) determines the electron spin relaxation time, whereas the second one the position-dependent nuclear spin relaxation time. The generalization to the case of arbitrary nuclear spin $I$ is straightforward if we consider that the nuclear spin flips probabilities $W_{-+}\left(\mathbf{r}_{n}\right)$ and $W_{+-}\left(\mathbf{r}_{n}\right)$ describe transitions between adjacent nuclear spin levels.

In view of Eq. (2.8) the definitions of the electronic and nuclear spin relaxation times should be considered with some caution, as an exponential decay for the electronic magnetization is obtained only for the case when the nuclear population approaches equilibrium, or is kept at a fixed nonequilibrium value. For the latter case a new kinetic equilibrium value for the electron polarization, $\tilde{D}_{0}$, will be reached, which is determined by the value for $D$ that makes $d D / d t=0$ in Eq. (2.8) for the constant nonequilibrium nuclear polarization $\Delta\left(\mathbf{r}_{n}\right)$. For calculating the nuclear spin relaxation time we use the total spin angular momentum conservation

$$
\frac{d D}{d t}=\frac{2 I(I+1)(2 I+1)}{3} \sum_{n} \frac{d \Delta\left(\mathbf{r}_{n}\right)}{d t}
$$

assume the electron spin polarization is refreshed, and so remains approximately a constant, and separate the resulting equation into $n$ equations: one for each nucleus.

For the case of a two dimensional (2D) system, with a constant density of states, $N_{e}(\varepsilon)=m_{e} / 2 \pi\left(m_{e}\right.$ is the electron mass), the electronic and nuclear relaxation times are

$$
T_{1 e}^{-1}=\frac{1}{A} \sum_{n} \frac{512 \pi^{2} m_{e} \beta_{e}^{2} \beta_{n}^{2}\left|\psi\left(\mathbf{r}_{n}\right)\right|^{4}}{9 \hbar^{3}(2 I+1)}
$$

and

$$
T_{1 n}^{-1}\left(\mathbf{r}_{n}\right)=\frac{128 \pi m_{e} \beta_{e}^{2} \beta_{n}^{2}\left|\psi\left(\mathbf{r}_{n}\right)\right|^{4} k_{B} T}{3 \hbar^{5} I(I+1)(2 I+1)},
$$


where $A$ represents the area of the $2 \mathrm{D}$ system. Temperature corrections to these times are of the order of $k_{B} T / E_{F}, E_{F}$ being the Fermi energy of the electronic system. As we can see from Eq. (2.10) the electronic spin relaxation time is temperature independent (up to the first order of approximation) suggesting that the relaxation mechanism due to the hyperfine interaction can be the dominant one at low temperatures, as the relaxation times corresponding to other mechanisms increase as the temperature decreases.[14] According to Eq. (2.11) the nuclear spin relaxation time follows the well known Korringa law, which states that the ratio $1 /\left(T_{1 n} T\right)$ is temperature independent.

Let us turn our attention to the quasi-two-dimensional case of a QW. In such systems the growth-direction electronic motion is restricted to discrete energy levels through confinement in a potential well. Accordingly, the electron energy dispersion can be written as $\varepsilon(\mathbf{k})=\varepsilon_{i}+\mathbf{k}^{2} / 2 m_{e}$, where $\varepsilon_{i}$ is the minimum of the conduction subband $i$ and $\mathbf{k}$ is the electron momentum in the plane of the quantum well. If the separation between the energy levels is large enough compared to the Fermi energy, the electrons will be frozen in the first energy subband leading to the practical realization of a $2 \mathrm{D}$ electronic system. In this case the electronic and nuclear spin relaxation times are given by Eqs. (2.10) and (2.11). However, if the Fermi energy exceeds the separation between the energy levels, we must consider a multiply-occupied subband system. For the case of multiple subband occupancy the density of states is

$$
N(\varepsilon)= \begin{cases}m_{e} / 2 \pi, & \varepsilon_{1}<E_{F}<\varepsilon_{2} \\ 2 m_{e} / 2 \pi, & \varepsilon_{2}<E_{F}<\varepsilon_{3} \\ \cdots & \\ l m_{e} / 2 \pi, & \varepsilon_{l}<E_{F}<\varepsilon_{l+1}\end{cases}
$$

where we consider that the $l$-th is the last subband of the system occupied by electrons. Two additional complications arise that prevent direct use of this larger density of states in Eqs. (2.10-2.11). First, the envelope functions for the different subbands will differ, and second, electron spin-flip scattering can take place inside the same subband or between two different subbands.

The electron and nuclear spin relaxation times for a QW can still be written very simply, and physically, in terms of the electronic local density of states,

$$
T_{1 e}^{-1}=\frac{1}{V} \sum_{n} \frac{1024 \pi^{3} \beta_{e}^{2} \beta_{n}^{2} \int d \varepsilon A_{e}^{2}\left(\mathbf{r}_{n}, \varepsilon\right) f_{F D}^{\prime}(\varepsilon)}{9 \hbar(2 I+1) \int d \mathbf{r} d \varepsilon A_{e}(\mathbf{r}, \varepsilon) f_{F D}^{\prime}(\varepsilon)}
$$

and

$$
T_{1 n}^{-1}\left(\mathbf{r}_{n}\right)=\frac{512 \pi^{3} \beta_{e}^{2} \beta_{n}^{2} k_{B} T \int d \varepsilon A_{e}^{2}\left(\mathbf{r}_{n}, \varepsilon\right) f_{F D}^{\prime}(\varepsilon)}{3 \hbar I(I+1)(2 I+1)} .
$$

The electronic local density of states at the nuclear position $\mathbf{r}_{n}$ is

$$
A_{e}\left(\mathbf{r}_{n}, \varepsilon\right)=\sum_{m}\left|\psi_{m}\left(\mathbf{r}_{n}\right)\right|^{2} \delta\left(\varepsilon-E_{m}\right),
$$

where $m$ labels the state, $E_{m}$ its energy, and $\psi_{m}\left(\mathbf{r}_{n}\right)$ its wavefunction at the $n$ 'th nucleus.

In general for any QW structure the dispersion relation is quasi-two-dimensional, therefore, the electronic wave function will be written as a product between an envelope function, $\phi(z)$, and a Bloch function, $u_{n \mathbf{K}}(\mathbf{r})$, such that $\psi_{\mathbf{K}, n}\left(\mathbf{r}_{n}\right)=\exp [i \mathbf{K} \cdot \mathbf{R}] \phi(z) u_{n \mathbf{K}}\left(\mathbf{r}_{n}\right)$, and

$$
A_{e}\left(\mathbf{r}_{n}, \varepsilon\right)=\sum_{j \mathbf{k}}\left|\psi_{j \mathbf{K}}\left(\mathbf{r}_{n}\right)\right|^{2} \delta\left(\varepsilon-E_{j \mathbf{K}}\right),
$$

where $j$ is the subband index and $\mathbf{K}$ the momentum. The envelope functions for parabolic quantum wells will be evaluated both analytically and numerically. For the numerical estimations we will use a $\mathbf{k} \cdot \mathbf{p}$ calculation.[15] For the QW's stepwise density of states,

$$
\begin{aligned}
& \int d \varepsilon A_{e}^{2}\left(\mathbf{r}_{n}, \varepsilon\right) f_{F D}^{\prime}(\varepsilon) \\
& =\left|u\left(\mathbf{r}_{n}\right)\right|^{4} \sum_{j, k}\left|\phi_{j}\left(\mathbf{r}_{n}\right)\right|^{2}\left|\phi_{k}\left(\mathbf{r}_{n}\right)\right|^{2} \Theta\left(\varepsilon_{\max \{j, k\}}\right),
\end{aligned}
$$

with $\Theta\left(\varepsilon_{j}\right)$ a temperature dependent factor. Such factors are important when the Fermi energy approaches an $\varepsilon_{i}$, i.e., when the electrons start to occupy higher conduction subbands. The energy integration over the step-like density of states in Eq. (2.17) leads to temperature dependent factors of the following form:

$$
\Theta\left(\varepsilon_{j}\right)=\frac{1}{\exp \left[\frac{\varepsilon_{j}-E_{F}}{k_{B} T}\right]+1} .
$$

Eq. (2.18) clarifies the role of the temperature when higher subbands start to be occupied, i.e., the Fermi energy is of the order of $\varepsilon_{i}$.

For $\mathbf{K} \sim 0$ the value of the Bloch function for the conduction band can be approximated as a constant. The approximate constant value of the Bloch function at the ${ }^{71}$ Ga nucleus site, $\left|u\left(\mathbf{r}_{n}\right)\right|^{2}=5.2 \times 10^{25} \mathrm{~cm}^{-3}$, was extracted by comparing our numerically calculated nuclear relaxation time with the available experimental data for a GaAs $/ \mathrm{Al}_{0.1} \mathrm{Ga}_{0.9} \mathrm{As}$ QW.[16]

\section{THE PARABOLIC QUANTUM WELL}

In this section we will discuss the nuclear spin relaxation time for the case of parabolic QW's. The special form of the confining potential in these structures allows for exact analytical results which permit an evaluation of the nuclear spin relaxation time. The analytical results will be compared with numerical data obtained from the $\mathbf{k} \cdot \mathbf{p}$ calculation.

An initial nuclear polarization obtained by DNP will be inhomogeneous, and for short times will be proportional to $T_{1 n}^{-1}(z)$, so for one occupied subband the initial nuclear magnetization

$$
m(z, t=0) \propto|\phi(z)|^{4} .
$$


Our calculation assumes that DNP is performed in the absence of an applied electric field and for single subband occupancy. In this case $\phi(z)$ corresponds to electrons situated in the first subband of the conduction band. The value of the nuclear magnetization will be influenced mainly by two processes, spin relaxation and spin diffusion. We track the evolution of the position dependent nuclear polarization in order to obtain the time dependence of the total nuclear magnetization in the PQW. Considering both spin diffusion and spin relaxation, the total nuclear magnetization across the PQW can be evaluated as the solution to the following equation:

$$
\frac{d m(z, t)}{d t}=D \frac{\partial^{2} m(z, t)}{\partial z^{2}}-\frac{m(z, t)}{T_{1 n}(z)}
$$

where $D$ represents the nuclear diffusion constant. The combined effects of nuclear spin diffusion and relaxation indicate that the longer-time dynamics of the magnetization is non-exponential in PQW's.[16] If we consider that initially the PQW is in a state where only the first conduction band is filled with electrons, during the DNP procedure nuclei situated close to the sample's center become strongly polarized. The initial polarization is smaller for nuclei farther away from the center of the PQW where the electronic density is smaller. Even farther from the PQW center, where the electronic wave function is essentially zero, the nuclei are initially unpolarized. However, nuclear spin diffusion can bring the polarization generated in the center of the well out to the entire sample.

The position dependent initial nuclear spin relaxation time can be extracted from Eq. (3.2) if the nuclear diffusion term is absent $(D=0)$. As a consequence the observed total initial nuclear relaxation time is given by

$$
\frac{1}{T_{1 n}}=\frac{\int d z T_{1 n}^{-1}(z) \mathcal{P}_{n}(z)}{\int d z \mathcal{P}_{n}(z)}
$$

where $\mathcal{P}_{n}(z)$ is proportional to the initial positiondependence of the nuclear polarization. For the case of optical pumping, where the nuclear polarization is realized through the hyperfine interaction, $\mathcal{P}_{n}(z) \propto\left|\phi_{1}(z)\right|^{4}$. Our estimation of the nuclear spin relaxation times will be done for temperatures where DNP in GaAs is efficient ( $T \sim 30 \mathrm{~K})$, however, we expect that the obtained results can be easily extended to higher temperatures.

\section{A. Analytical Results}

In an ideal parabolic QW (IPQW) the form of the confining potential is

$$
V(z)=\frac{1}{2} m_{e} \omega_{0}^{2} z^{2},
$$

where $\omega_{0}$ is the characteristic frequency. The electronic envelope functions and the characteristic subband energies are obtained from the time-independent Schrodinger equation

$$
\left(-\frac{\hbar^{2}}{2 m_{e}} \frac{d^{2}}{d z^{2}}+\frac{1}{2} m_{e} \omega_{0}^{2} z^{2}\right) \phi(z)=\varepsilon_{n} \phi(z),
$$

as

$$
\phi_{n}(z)=N_{n} \exp \left[-\frac{m_{e} \omega_{0}}{2 \hbar} z^{2}\right] H_{n-1}\left[\left(\frac{m_{e} \omega_{0}}{\hbar}\right)^{1 / 2} z\right]
$$

and

$$
\varepsilon_{n}=\left(n-\frac{1}{2}\right) \hbar \omega_{0}
$$

where the subband index $n$ is a natural number, $H_{n}(x)$ are the Hermite polynomials, and $N_{n}$ is the normalization factor which can be obtained analytically if the width of the QW is much bigger than the PQW length scale $L \gg l_{0}\left(l_{0}=\sqrt{\hbar /\left(m_{e} \omega_{0}\right)}\right)$. According to Eq. (3.7) the energy distance between adjacent conduction subbands, $\Delta E=\varepsilon_{n}-\varepsilon_{n-1}$, is a constant depending only on the confining potential's characteristic frequency $\omega_{0}$.

An additional external electric field, $\mathcal{F}$, will change the Schrodinger equation by introducing a field dependent term of the form

$$
V(z)=e \mathcal{F} z,
$$

whose effects can be easily understood if absorbed into the parabolic confining potential. The external electric field will not change the form of the envelope functions, however their positions in the QW are shifted by a distance proportional to the strength of the field, $z_{0}=e \mathcal{F} / m_{e} \omega_{0}^{2}$. The new wave functions are

$$
\begin{aligned}
\phi_{n}(z, \mathcal{F})= & N_{n} \exp \left[-\frac{m_{e} \omega_{0}}{2 \hbar}\left(z-z_{0}\right)^{2}\right] \\
& \times H_{n-1}\left[\left(\frac{m_{e} \omega_{0}}{\hbar}\right)^{1 / 2}\left(z-z_{0}\right)\right] .
\end{aligned}
$$

The subband energy in the presence of an external electric field will be lowered to a value

$$
\varepsilon_{n}(\mathcal{F})=\varepsilon_{n}-\frac{e^{2} \mathcal{F}^{2}}{2 m_{e} \omega_{0}^{2}},
$$

where the energy difference, $\Delta E=\varepsilon_{n}-\varepsilon_{n-1}$, is the same as in the absence of the electric field. The electric field will allow a direct control of the electron density profile across the sample leading to the manipulation of the nuclear spin relaxation times in PQW's.

In the following we will consider two different PQW configurations, i.e, two structures with different characteristic frequencies $\omega_{0}$ : the first structure will be referred to as IPQW I $\left(\omega_{0}=2.3 \times 10^{13} \mathrm{~s}^{-1}, \Delta E=15 \mathrm{meV}\right)$ and the second one as IPQW II $\left(\omega_{0}=7.6 \times 10^{13} \mathrm{~s}^{-1}, \Delta E=50\right.$ $\mathrm{meV})$. This choice of the PQW's will allow us to discuss the relative influences of temperature, electric field, and well structure on the nuclear spin relaxation times. 

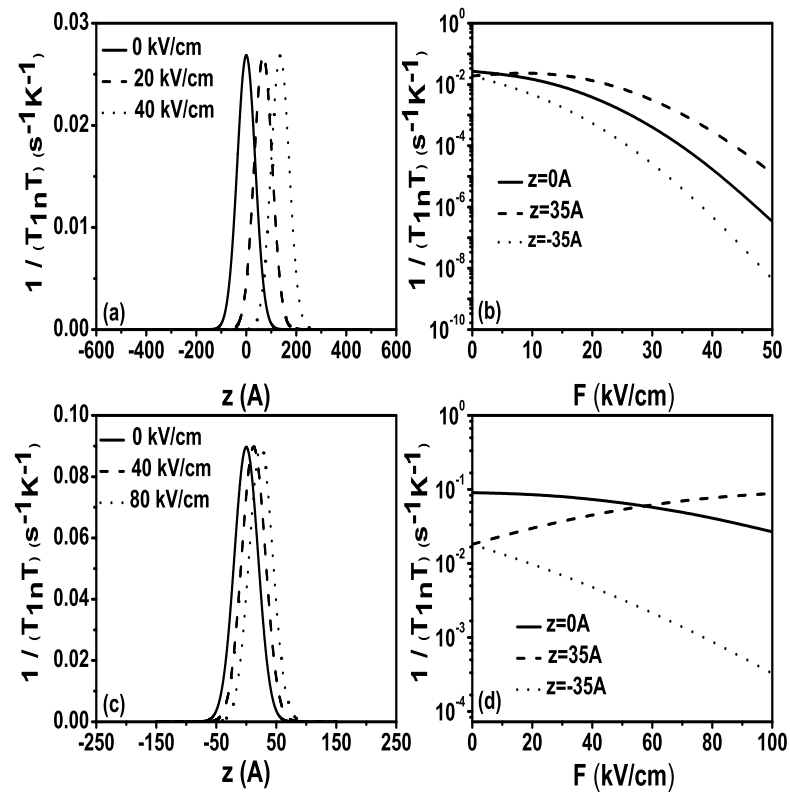

FIG. 1: The position dependence of $1 /\left(T_{1 n} T\right)$ for different values of the external electric field [(a) IPQW I and (c) IPQW II]. The field dependence of the $1 /\left(T_{1 n} T\right)$ ratio for $\delta$-doped layers situated at different positions across the QW [(b) IPQW I and (d) IPQW II].

In Fig. 1(a) and 1(c) we present the position dependence of $1 /\left(T_{1 n} T\right)$ as a function of the applied field for the case of single subband occupancy. IPQW I is more suitable than IPQW II for electric field control, as the rate of change with field manifest in $1 /\left(T_{1 n} T\right)$ is much larger. The displacement of the electronic wave function with an applied electric field is larger for wells with smaller values of the characteristic frequency, as $z_{0} \sim \omega_{0}^{-2}$, and IPQW I has a smaller $\omega_{0}$ than IPQW II. In Fig. 1(b) and 1(d) we plot the value of the $1 /\left(T_{1 n} T\right)$ ratio as a function of the applied electric field for three different positions in the QW. These positions corresponded to initial nuclear polarization via DNP of the maximal value (center of the well) and half of the maximal value. The tunability of the nuclear spin relaxation times at these particular position is a few orders of magnitude, with larger tunability for IPQW I.

This property of the PQW's will allow direct control of the relaxation process in $\delta$-doped layers of nuclei situated at certain positions inside the QW. The only requirement is that the $\delta$-doped layer is situated in a region where the DNP procedure will produce a high enough initial polarization of the nuclei. The nuclei inside these layers will not be affected by nuclear diffusion, as this process requires similar nuclear species, or at least nuclear species with close values of the resonance frequencies.[11]

In Fig. 2(a) and 2(c) we present $1 /\left(T_{1 n} T\right)$ for uniformly distributed nuclei as a function of the Fermi energy (which depends on the electron density). Manipu-
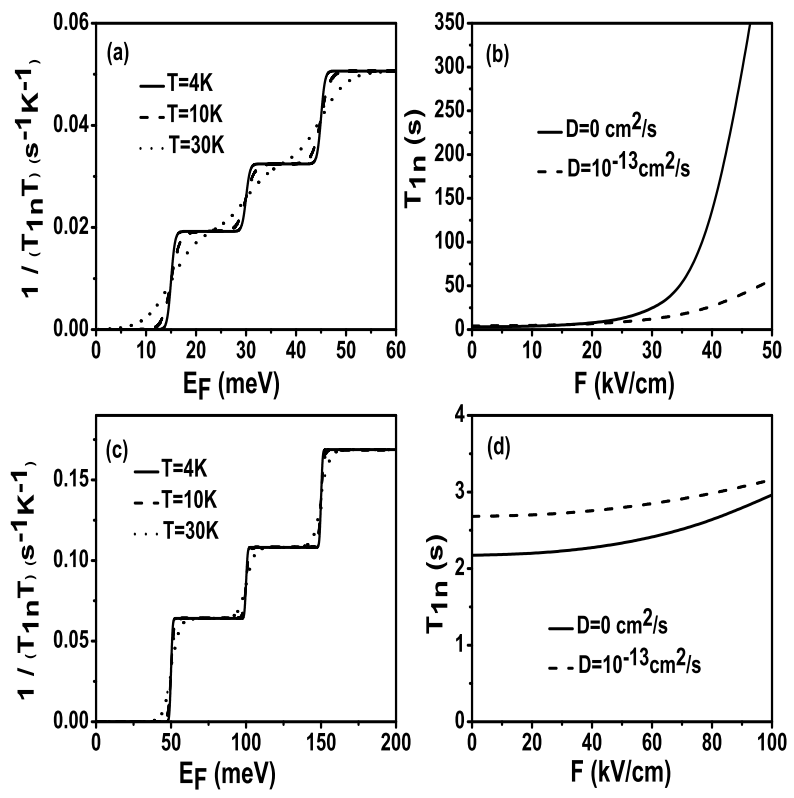

FIG. 2: The initial value of $1 /\left(T_{1 n} T\right)$ as a function of Fermi energy at several temperatures [(a) IPQW I and (c) IPQW II]. The electric field dependence of the initial nuclear spin relaxation times for single subband occupancy, with and without nuclear spin diffusion [(b) IPQW I and (d) IPQW II].

lation of the electronic density in the QW can be accomplished with a gate. The reference value for the energy is set at $-\Delta E$ below the minimum of the first conduction band. $1 /\left(T_{1 n} T\right)$ depends stepwise on the Fermi energy at very low temperatures, increasing every time a new conduction electron subband becomes occupied. However corrections to the Korringa law become important at higher temperatures for Fermi energies near these steps. As we can see from Fig. 2(a) at $\mathrm{T}=30 \mathrm{~K}$ for IPQW I, temperature-induced smearing will destroy the stepwise character of $1 /\left(T_{1 n} T\right)$. For IPQW II due to a larger energetic separation between subbands the stepwise behavior remains for higher temperatures.

Fig. 2(b) and 2(d) present the initial value of the nuclear spin relaxation time as a function of the applied electric field. The relaxation time, both in the absence of and presence of nuclear diffusion, is extracted as the first derivative of the total nuclear magnetization in the QW. For a nuclear spin diffusion constant close to the one extracted from observations in bulk GaAs systems, the diffusion process will diminish significantly the electric field tunability of the nuclear spin relaxation time. Due to the larger distances the electronic wave function is displaced in IPQW I, the tuning calculated there is significantly more robust to nuclear spin diffusion than IPQW II.

Thus we see that different PQW structures behave in 

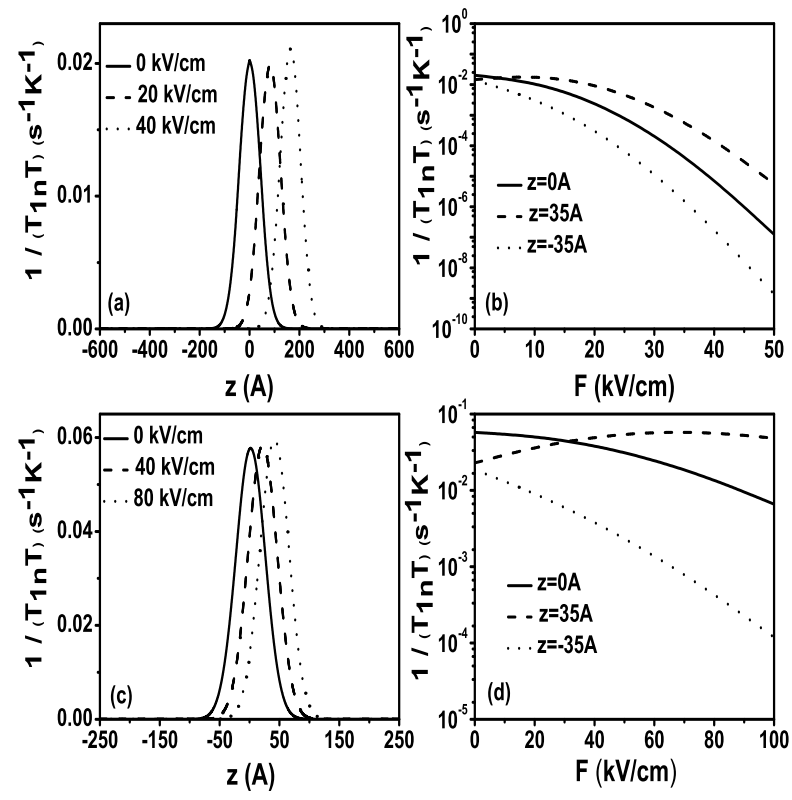

FIG. 3: The position dependence of $1 /\left(T_{1 n} T\right)$ for different values of the external electric field [(a) PQW I and (c) PQW II]. The field dependence of $1 /\left(T_{1 n} T\right)$ for $\delta$-doped layers situated at different position across the QW [(b) PQW I and (d) PQW II].

a complementary fashion in the presence of external electric fields. If the electric field is used as an electron density gate, controlling the subband occupancy in the system, the stepwise dependence of $1 /\left(T_{1 n} T\right)$ on electron density can be better observed in structures with a larger splitting of the energy levels, for larger subband separations suppress the temperature dependence of the steps. On the other hand, if the electric field is used to tune the nuclear spin relaxation time by controlling the electronic local density of states (tilting the PQW), structures with a smaller value of the characteristic frequency, and implicitly with a smaller splitting of the energy subbands, are more resilient to nuclear diffusion. When the field is used to tilt the PQW, inserting a $\delta$-doped layer of a different material at a certain position across the $\mathrm{QW}$ will permit sensitive control of the relaxation time over a few orders of magnitude (see Fig 1 (b) and (d)). The $\delta$-doped layer should be made of a different material, with a different NMR resonance frequency than the host material.

\section{B. Numerical Results}

In this section we will numerically evaluate nuclear spin relaxation times for more realistic versions of the PQW's. The first structure (referred from now on as PQW I) is a $1000 \AA \mathrm{Al}_{x} \mathrm{Ga}_{1-x}$ As system sandwiched between two $100 \AA \mathrm{Al}_{0.4} \mathrm{Ga}_{0.6} \mathrm{As}$ barriers. [7] The value of the aluminum concentration across the QW varies from
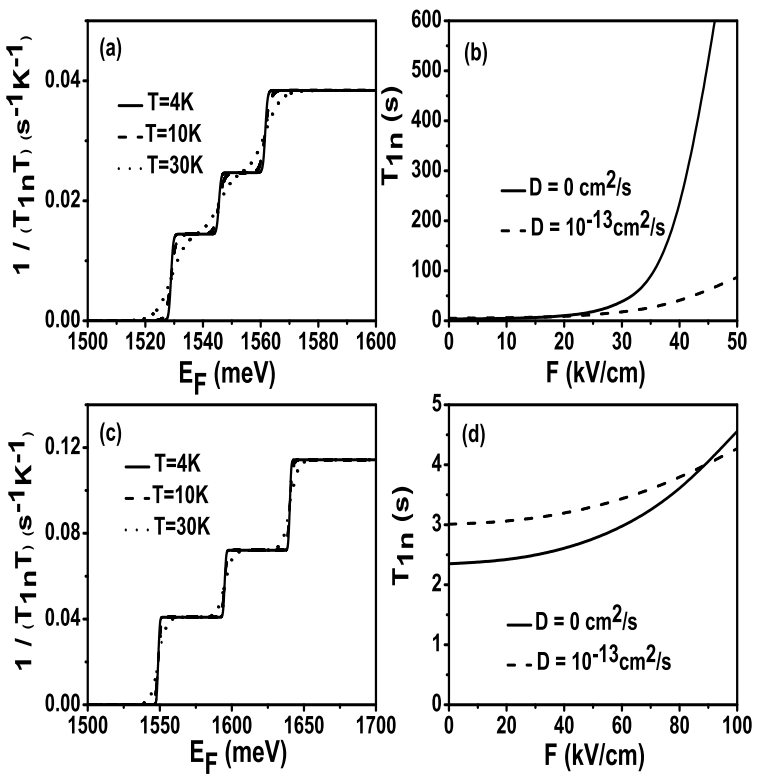

FIG. 4: The initial value of $1 /\left(T_{1 n} T\right)$ as a function of Fermi energy at several temperatures [(a) PQW I and (c) PQW II]. The electric field dependence of the initial nuclear spin relaxation times for single subband occupancy, with and without nuclear spin diffusion [(b) PQW I and (d) PQW II].

0.4 in the barriers to 0 in the QW's center. The second structure (referred from now on as PQW II) is a 300 $\AA \mathrm{Al}_{x} \mathrm{Ga}_{1-x} \mathrm{As}$ system sandwiched between two $100 \AA$ $\mathrm{Al}_{0.3} \mathrm{Ga}_{0.7} \mathrm{As}$ barriers. [6] In this case the aluminum concentration across the sample varies from 0.3 in the barriers to 0 in the center of the QW. Our estimation of the energy difference between the first and second conduction bands is $\Delta E=16.6 \mathrm{meV}$ for $\mathrm{PQW} \mathrm{I}$ and $\Delta E=46.1 \mathrm{meV}$ for PQW II, values which are close to the ones considered in the two analytic examples. The evaluation of the envelope functions in these particular structures is done based on a 14-band multilayer $\mathbf{k} \cdot \mathbf{p}$ calculation. Therefore additional effects will be evident in the $\mathbf{k} \cdot \mathbf{p}$ calculations, including a diminished value of the energy difference between higher conduction subbands.

In Fig. 3(a) and 3(c) we present the position dependence of $1 /\left(T_{1 n} T\right)$ for different values of the applied electric field. As seen in the analytic studies, in PQW's the applied electric field leads to a displacement of the electronic wave function with direct implications for the position dependent nuclear spin relaxation time. Fig. 3(b) and $3(\mathrm{~d})$ showcase the field dependence of $1 /\left(T_{1 n} T\right)$ for three different position across the QW's. These positions were selected in the same way as those for Fig. 1, corresponding to maximal and $50 \%$ of maximal initial nuclear polarization. The electric field tunability is much higher in PQW I, where the effects of the electric field are considerable even at low fields. The achieved tunability is a few orders of magnitude for both structures for any 
of the three considered positions. The results shown in Fig. 3 are very similar to those obtained with the analytic model and shown in Fig. 1. In Fig. 4 we present the value of $1 /\left(T_{1 n} T\right)$ for uniformly distributed nuclei as a function of the Fermi energy. Just as for the analytic results presented in Fig. 2 the temperature-induced smearing is more significant for PQW I, which has a smaller subband separation, than for PQW II. However the effects of nuclear diffusion are more pronounced for PQW I than for PQW II.

\section{SUMMARY}

We have performed a detailed analysis, both analytically and numerically, of the nuclear spin dynamics as a result of the hyperfine interaction in parabolic quantum wells. Analytical formulae for the electron and nuclear spin relaxation times were obtained for general 2D structures and extended for quasi 2D structures. The electronic structure enters these formulae in the form of the electronic local density of states. For the quasi 2D situation we considered an ideal parabolic QW, for which analytic results are available for the field-dependent envelope functions. Thus we were able to explore the electricfield manipulation of the nuclear spin relaxation time entirely within an analytic model. These results were checked with numerical $\mathbf{k} \cdot \mathbf{p}$ calculations for the envelope functions. The results obtained from analytical and numerical calculations are consistent, with small differences originating from approximations used in the analytical calculation.

Our specific calculations were for two different PQW structures, where the main difference was the value of the characteristic frequency in the confining potential (or subband energy separation). Electrical field control of the nuclear spin dynamics in PQW structures can be achieved in two different ways: by controlling the electronic subband occupancy or by controlling the position dependent electronic wave functions. When the electronic Fermi energy is changed a stepwise dependence of the nuclear spin relaxation time is predicted. Our calculation identifies the PQW with a larger energy splitting between subbands as more suitable for such manipulation. On the other hand, for single subband occupancy, a position dependent control of the nuclear spin relaxation time is possible as the electronic local density of states across the PQW is tunable by tilting the PQW potential with an electric field. In this case we suggested that the insertion of a $\delta$-doped layer of different NMR active nuclei than the host nuclei would allow manipulation of the nuclear spin relaxation time on the scale of a few orders of magnitude. Here samples with a small value of the energy splitting between adjacent electronic subbands are more suitable. The effect of nuclear spin diffusion on the nuclear spin dynamics for single subband occupancy was considered for several values of the applied electric field. We find that the presence of spin diffusion will diminish the electrical field control of the relaxation process. However, for the PQW with a smaller energy splitting of the subbands, electric field tunability of the nuclear spin relaxation time is more robust.

\section{Acknowledgments}

We would like to acknowledge stimulating discussions with D. D. Awschalom, W. H. Lau, D. Loss, A. V. Khaetskii, J. M. Kikkawa and J. M. Tang. This work was supported by DARPA/ARO DAAD19-01-1-0490.
[*] Permanent address: Department of Theoretical Physics, "Babeş-Bolyai" University of Cluj, 3400 Cluj, Romania.

[1] J. M. Kikkawa and D. D. Awschalom, Phys. Rev. Lett. 80, 4313 (1998).

[2] Semiconductor Spintronics and Quantum Computation, edited by D. D. Awshalom, N. Samarth, and D. Loss (Springer Verlag, Berlin, 2002).

[3] A. Berg, M. Dobers, R. R. Gerhardts, and K. von Klitzing, Phys. Rev. Lett. 64, 2563 (1990).

[4] S. E. Barrett, R. Tycko, L. N. Pfeiffer, and K. W. West, Phys. Rev. Lett. 72, 1368 (1994).

[5] J. H. Smet, R. A. Deutschmann, F. Ertl, W. Wegscheider, G. Abstreiter, and K. von Klitzing, Nature 415, 281 (2002).

[6] R. C. Miller, A. C. Gossard, D. A. Kleinman, and O. Munteanu, Phys. Rev. B 29, 3740 (1984).

[7] G. Salis, Y. Kato, K. Ensslin, D. C. Driscoll, A. C. Gossard, and D. D. Awschalom, Nature 414, 619 (2001).

[8] J. A. Marohn, P. J. Carson, J. Y. Hwang, M. A. Miller, D. N. Shykind, and D. P. Weitekamp, Phys. Rev. Lett. 75, 1364 (1995).
[9] A. Malinowski and R.T. Harley, Solid State Commun. 114, 419 (2000).

[10] G. Salis, D. T. Fuchs, J. M. Kikkawa, D. D. Awschalom, Y. Ohno, and H. Ohno, Phys. Rev. Lett. 86, 2677 (2001).

[11] A. Abragam, The Principles of Nuclear Magnetism (Oxford University Press, Oxford, 1961).

[12] J. Strand, B. D. Schultz, A. F. Isakovic, C. J. Palmstrom, P. A. Crowell, Phys. Rev. Lett. 91, 036602 (2003).

[13] D. Paget, Phys. Rev. B 25, 4444 (1982).

[14] A. W. Overhauser, Phys. Rev. 89, 689 (1953).

[15] J. T. Olesberg, Ph.D. Thesis, The University of Iowa (1999), unpublished.

[16] I. Țifrea and M. E. Flatté, Phys. Rev. Lett. 90, 237601 (2003). 